\title{
Rheumafaktoren und Immunglobuline als Störquellen bei einem heterogenen Enzym-Immunoassay
}

\author{
Von R. Allner \\ Zentrallabor der Städt. Kliniken Fulda, Lehrkrankenhaus der Philipps-Universität Marburg
}

(Eingegangen am 16. April/28. August 1984)

\begin{abstract}
Zusammenfassung: Die in dieser Arbeit vorgelegten Untersuchungsergebnisse wurden mit dem EnzymunTest ${ }^{\circledR}$ Digoxin an Modellproben erhalten. Die Modellproben setzten sich in ihrer Matrix aus entweder phosphatgepufferter 0,15 mol/1 Natriumchloridlösung pH 7,2 und einem Zusatz von Albumin bzw. Immunglobulinen oder aus verschiedenen Mengenverhältnissen von Albumin und im überwiegenden Anteil immunelektrophoretisch charakterisierter Immunglobulinspezifitäten zusammen. Steigende Mengen von Albuminen oder Immunglobulinen in den Modellproben täuschten entsprechend steigende Mengen Digoxin vor. Bei einem Anstieg bestimmter Immunglobulinspezifitäten in den Modellproben, auch wenn der Gesamtgehalt an Proteinen durch kompensatorische Maßnahmen unverändert blieb, nahm die Vortäuschung von Digoxin entsprechend zu.
\end{abstract}

Auch Rheumafaktoren können den Test in gleicher Weise stören.

Rheumatoid factors and immunoglobulins as interfering factors in an heterogeneous enzyme immunoassay

Summary: Enzymun-Test ${ }^{\circledR}$ Digoxin was used to analyse model samples. The matrix of the model samples consisted of either phosphate-buffered $0.15 \mathrm{~mol} / 1$ sodium chloride solution $\mathrm{pH} 7.2$ with addition of albumin or immunoglobulins, or of various ratios of albumin and immunoglobulins, most of which had been characterized immunoelectrophoretically. Increasing amounts of albumin or immunoglobulins in the model samples caused a simulated increase in the measured amount of digoxin. Apparent digoxin values also showed corresponding false increases when immunoglobulins of certain specificities were increased in the model samples, even when total protein was kept constant.

Rheumatoid factors showed similar interference.

\section{Einfuihrung}

Meßergebnisse enzymimmunologischer Tests stehen gelegentlich nicht im Einklang mit dem sogenannten „,klinischen Eindruck“. Entsprechende Beobachtungen gaben die Anregung zur vorliegenden Arbeit. Bei der Suche nach Störquellen im Untersuchungsmaterial mit Auswirkungen auf heterogene enzymimmunologische Verfahren wurde der EnzymunTest $^{\circledR}$ Digoxin eingesetzt.

Enzymimmunologische Verfahren sind Vergleichsmessungen. Zur Erstellung von Bezugskurven dienen Standardproben. Die Matrix der Standardpro- ben, insbesondere ihr Proteingehalt, entspricht weitgehend der Zusammensetzung des Serums gesunder Personen. Damit wird versucht, unspezifische Reaktionen, die zur Verfälschung der Meßwerte führen, soweit sie durch den Proteingehalt des Untersuchungsgutes ausgelöst werden, über diesen Weg der Ergebnisermittlung zu kompensieren. Trotz der Vielfältigkeit der Proteinspezifitäten im menschlichen Serum und daraus sich ergebender Störmöglichkeiten, gelingt dies in der Praxis auch weitgehend. Es ist aber denkbar, daß bei einer wesentlichen Abweichung der Proteinmuster von der Norm bei solchen Patientenseren der Kompensationsme- 
chanismus über die Bezugskurve nicht mehr ausreichend möglich ist. Es sei an Erkrankungen mit Dysproteinämie oder monoklonaler Gammopathie erinnert. Würden im Verlauf solcher Erkrankungen auch Proteinspezifitäten mit besonderer Bindungsaffinität zum wandständigen Antikörper oder zum Material des Reaktionsgefäßes gebildet, wären Störungen des immunologischen Reaktionsschrittes im Testverfahren mit entsprechenden Auswirkungen auf das Testergebnis unausweichlich.

Die immunchemischen Eigenschaften der Antigammaglobulinaktivität, die mit dem Begriff Rheumafaktoren umschrieben werden, sind hinlänglich bekannt. Für alle Rheumafaktoren charakteristisch ist ihre Affinität zur Fc-Region von Immunglobulinen der Klasse G (IgG) (1). Das natürliche Substrat ist zwar humanes Immunglobulin, doch sind auch Kreuzreaktionen mit tierischen $\gamma$-Globulinen bekannt, wenn auch die Affinität zu IgG animalischer Herkunft üblicherweise gering ist $(2,3)$. Immunkomplexe, wie unspezifische IgG-Aggregate oder an Festphasen gebundene $\gamma$-Globuline, kommen als Substrat gegenüber der Rheumafaktor-Aktivität in Betracht. Den Rheumafaktoren kommt aus diesen Gründen im enzymimmunologischen Test als Störquelle eine besondere Bedeutung zu. Im Hinblick auf Rheumafaktorinterferenz besonders eindringlich untersucht ist der Rubella-spezifische IgM-Nachweis (4). Auch im heterogenen enzymimmunologischen Test zur Digoxinbestimmung (Enzymun-Test ${ }^{\circledR}$ Digoxin) kann der Rheumafaktor als Störquelle in Betracht kommen.

In der vorliegenden Arbeit wird an Hand von Modellexperimenten versucht, die potentiellen Störquellen im menschlichen Serum aufzudecken. $\mathrm{Zu}$ diesem Zweck wurden aus Seren von Patienten mit Dysproteinämien, Gammopathien oder hohem Rheumafaktorgehalt mit geeigneten präparativen Methoden die der Störmöglichkeit verdächtigten Bestandteile isoliert und präparativ dargestellt. Die qualitative und quantitative Übertragbarkeit der Störquelle auf eine Albuminlösung wird als Beweis ihrer Existenz gewertet.

\section{Material und Methoden}

\section{Materialien}

Human-Albumin-Lösung $50 \mathrm{~g} / \mathrm{l}$ aus dem Programm des Herstellers Deutsches Rotes Kreuz Hessen.

Phosphatgepufferte $0,15 \mathrm{~mol} / \mathrm{l}$ Natriumchlorid-Lösung $\mathrm{pH} 7,2$ Behringwerke AG, Marburg, Best.-Nr.: $\varnothing$ SGS 44.

Digoxin, Reinsubstanz aus dem Programm der Firma E. Merck, Darmstadt, Best.-Nr.: 3054

\section{Untersuchungsmethoden}

RheumaTec Labordiagnostica Goedecke, Freiburg,

Enzymun-Test ${ }^{\circledR}$, Digoxin Immundiagnostica, Boehringer Mannheim, Best.-Nr.: 199656.

Absorption und Isolation des Rheumafaktprs erfolgte nach einer von Kolb (5) entwickelten Methode.

Humanes Immunglobulin wurde durch Ammoniumsulfat- bzw. Natriumsulfatfällung aus Serum von Einżelpersonen gewonnen (6-8).

Träger-Elektrophorese und Immunelektrophorese wurden zur Darstellung des Proteingehalts der Patientenseren, der HumanAlbumin-Lösung, der aus Patientenseren isolierten Immunglobulinfraktionen und der aus Human-Albumin und isolierten Immunglobulinfraktion hergestellten Modellproben eingesetzt. Dazu wurde ein Elektrophorese-Gerät der Firma Boskamp, Gerätebau, Hersel/Bonn benutzt. Als Träger dienten Acetatfolien der Firma Sartorius, Göttingen, die Anfärbung erfolgte mit Amidoschwarz. Zur Auswertung stand ein Scanner MR 693 mit Schreiber PR 693 der Firma Vogel, Gießen zur Verfügung. Zur Immunelektrophorese wurde Agarose-Gel IEP von Hyland DiagnosticaTravenol, Lap. Inc., Deerfield Illinois, USA, eingesetzt.

Zur quantitativen Bestimmung der in den Immunglobulinklassen vorhandenen Immunglobulinmengen wurde die Mancini-Technik (9) unter Verwendung von Diffusionsplatten Tri-Partigen ${ }^{\circledR}$ der Behringwerke Marburg, Best.-Nummer für IgA = OTDT 02; IgG = OTDS 02; IgM= OTDU 02 eingesetzt.

Protein wurde quantitativ nach der Methode von T. E. Weichselbaum (10) bestimmt.

Die quantitative Digoxinbestimmung erfolgte über Standardwerte. Die Berechnung der Standardkurve und der Probenergebnisse erfolgte unter Verwendung eines Hewlett-Packard Taschenrechners HP-67 nach der Funktionsbeschreibung $C=a_{0}+a_{1} \cdot 1 / E+$ $a_{2} \cdot 1 / E^{2}$. Für alle Berechnungen lagen Dreifachmessungen vor. Die photometrischen Messungen erfolgten am Digital-Photometer PCP der Firma Eppendorf.

\section{Arbeitsweise}

Die Untersuchungen zur Aufdeckung von Störquellen erfolgten an Modellproben in kleinen Serien (Tab. 2, 3, 4, 5). Die Modellproben bestanden aus Human-Albumin-Lösung oder einem Gemisch von Human-Albumin und aus Patientenseren isolierten Immunglobulinen. Für notwendige Verdünnungen wurde phosphatgepufferte Natriumchlorid-Lösung verwendet. Eine Serie bestand in der Regel aus fünf Einzelproben, wobei die Gesamtproteinmenge jeder Probe $50 \mathrm{~g} / \mathrm{l}$ betrug, die Proben sich selbst aber durch einen steigenden Anteil an zugesetżten Immunglobulinmengen unterschieden. Die zugesetzten Immunglobulinmengen werden folgendermaßen symbolisiert:

$$
\begin{aligned}
& \mathrm{a}=0 \mathrm{~g} / \mathrm{l} ; \\
& \mathrm{b}=15 \mathrm{~g} / \mathrm{l} ; \\
& \mathrm{c}=25 \mathrm{~g} / \mathrm{l} ; \\
& \mathrm{d}=35 \mathrm{~g} / \mathrm{l} ; \\
& \mathrm{e}=50 \mathrm{~g} / \mathrm{l} \text { (Tab. 2, 3, 4, 5). }
\end{aligned}
$$

Das pro Serie zur Aufstockung der Human-Albumin-Lösung eingesetzte Immunglobulinpräparat stammte jeweils nur von einem Patientenserum. Isoliert und angereichert wurden die Immunglobuline aus Seren von Patienten mit Dysproteinämie oder Gammopathie. Außerdem stand ein Serum mit hohem Rheumafaktorgehalt zur Verfügung. Die Blutentnahmen erfolgten stets morgens am liegenden Patienten vor der Nahrungsaufnahme. Die täglich angefallenen Seren wurden gesammelt und bei $-20^{\circ} \mathrm{C}$ verschlossen gelagert (max. 4 Monate). Einmal aufgetaute Seren wurden sofort zur Immunglobulinpräparation eingesetzt. Die Immunglobulinpräparate wurden nicht länger als sieben Tage im Kühl- 
schrank bei $+4^{\circ} \mathrm{C}$ gelagert. Die Patienten nahmen zur Zeit der Blutentnahme verschiedene Medikamente ein. Bekannte, dem Digoxin strukturverwandte und damit mit dem Antikörper des Testes kreuzreagierende Substanzen (12) haben die Patienten zur Probennahmezeit nicht eingenommen. Eine Verschleppung von Digoxin oder Digitoxin im Rahmen der Immunglobulinpräparation aus diesen Seren konnte durch geeignete Kontrolluntersuchungen ausgeschlossen werden. Die in den Tabellen 1, 2, 3, 4 und 5 aufgeführten Nummern (Nr.) identifizieren die Modellproben mit Immunglobulinzusatz aus der gleichen Serumprobe.

Das Serum zur Isolierung des Rheumafaktors nach der Methode von Kolb (5) täuschte mehr als 5 nmol/ Digoxin vor, der Patient selbst war ohne entsprechende Medikation. Der isolierte Rheumafaktor wurde in steigenden Titermengen pro Einzelprobe einem Serum mit bekanntem Digoxingehalt zugesetzt. Bei der $\mathrm{Be}-$ rechnung der Digoxinmengen wurden durch den Rheumafaktorzusatz in den einzelnen Proben entstandene Serumverdünnungen korrigiert.

Um einen Einblick über die Auswirkungen der Störungen auf den Digoxinnachweis zu erhalten, wurden alle Modellproben sowohl ohne als auch mit Digoxinzusatz untersucht. Dabei war der Digoxinzusatz zu den Proben innerhalb einer Serie stets gleich.

\section{Statistische Methoden}

Zur Charakterisierung der Wiederholbarkeit in der Serie wurde das arithmetische Mittel ( $\bar{x})$, die Standardabweichung $(\sigma)$ und der Variationskoeffizient (VK) bei Mehrfachbestimmungen ermittelt. Zur Beurteilung der Beeinflussung der Meßergebnisse durch den Immunglobulingehalt der Probe in den Untersuchungsserien wurde als mathematisch-statistische Interpretationshilfe der Korrelationskoeffizient und der $t$-Test für Korrelationen herangezogen. $\mathrm{Da}$ auf Grund der Versuchsanordnung Gruppen (a, b, c, d, e) mit gleichen Immunglobulinmengen gebildet werden können, ließen sich die vermuteten Abhängigkeiten der Meßwerte von der Immunglobulinmenge auch mit dem t-Test für Paar-Analysen nach Student (11) beschreiben.

\section{Qualitätskontrolle und Wiederfindung}

Bei Mehrfachbestimmungen in der Serie $(N=10)$ betrug der Variationskoeffizient (VK) $5,4 \%$, bei Wiederholungsuntersuchungen von Tag zu Tag 6,8\%. Bei Wiederfindungsversuchen mit Digoxinzusatz zur Probe $(\mathrm{N}=10)$ wurden $95 \%$ wiedergefunden. Die Nachweisgrenze für Digoxin liegt für den Enzymun-Test ${ }^{\oplus} \mathrm{Di}$ goxin bei 0,38 bis $0,64 \mathrm{nmol} / \mathrm{l}$.

\section{Ergebnisse}

Mit steigenden Albuminmengen in Modellproben, die aus einer phosphatgepufferten NatriumchloridLösung bestanden, kommt es zu einer Abnahme der Bindung enzymmarkierter Antigene, meßbar in einem Rückgang der Substratumsätze in der Indikatorreaktion (Abb. 1). Der Vorgang wiederholt sich in gleicher Weise in den Modellproben, denen in steigenden Mengen Immunglobuline zugesetzt wurden (Abb. 2). Die eingesetzten Immunglobuline stammten aus Seren gesunder Personen. Die Immunglobulinpräparate enthielten nach der TrägerElektrophorese kein Albumin, in der Immunelektrophorese fanden sich die drei Immunglobulinklassen: Immunglobulin A (IgA), Immunglobulin G (IgG) und Immunglobulin $M$ (IgM).

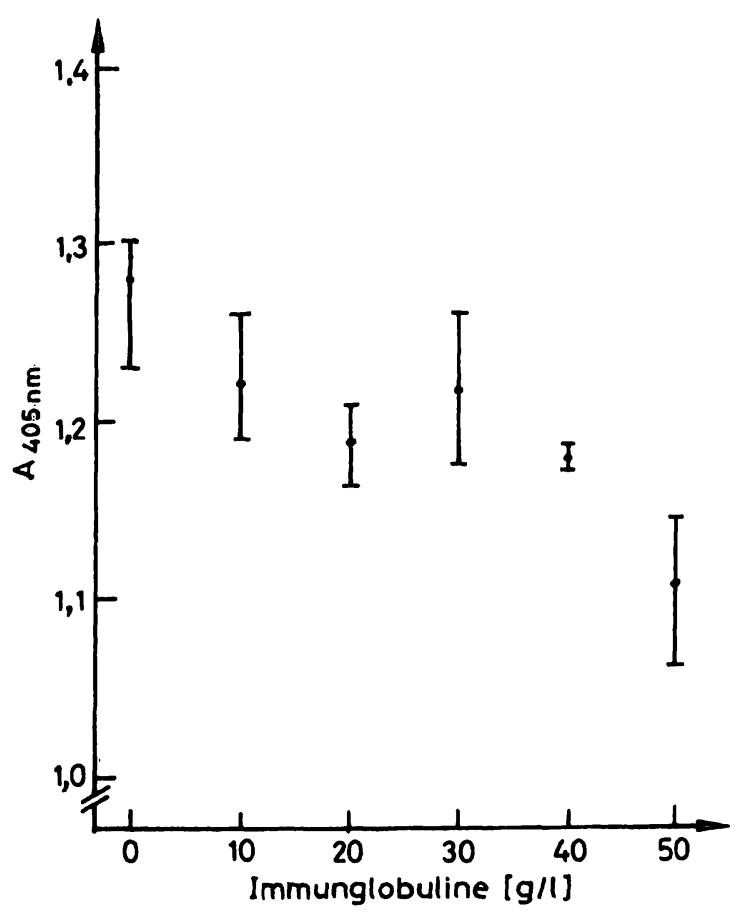

Abb. 2. Gemessene Änderungen der Absorbanz in der Digoxinbestimmung mil dem Enzymun-Test ${ }^{\star}$ Digoxin. Dic Proben bestanden aus phosphatgepufferter $0,15 \mathrm{~mol} / \mathrm{Na} \mathrm{Na}$ triumchloridlösung $\mathrm{pH}=7,2$, aufgestockt mit stcigenden Mengen von Immunglobulinen $(g / l)$. 
Tab. 1. Ergebnisse der quantitativen Bestimmung der Immunglobulinklassen (IgA, IgG und IgM) in Seren mit Gammopathie; Patientenidentifizierung nach Nummern (Nr.) $1-11$ in der Gruppe A und Nummern (Nr.) 1-6 in der Gruppe B. Immunelektrophoretische Identifizierung der Gammopathie nach Klassenzugehörigkeit und Leichtkettentypisierung (L-Ketten).

\begin{tabular}{|c|c|c|c|c|c|}
\hline \multirow[t]{3}{*}{$\mathrm{Nr}$} & \multicolumn{3}{|c|}{$\begin{array}{l}\text { Gammopathie } \\
\text { Klassenzugehörigkeit }\end{array}$} & \multirow[t]{3}{*}{ Klasse } & \multirow{3}{*}{$\begin{array}{l}\text { L-Ket- } \\
\text { ten-Typ }\end{array}$} \\
\hline & $\operatorname{Ig} A$ & IgG & IgM & & \\
\hline & $(g / l)$ & $(g / l)$ & $(g / l)$ & & \\
\hline
\end{tabular}

\begin{tabular}{|c|c|c|c|c|c|c|}
\hline \multicolumn{7}{|l|}{ A } \\
\hline & $\begin{array}{l}2 \\
3\end{array}$ & $\begin{array}{l}1,33 \\
0,48\end{array}$ & $\begin{array}{l}10,50 \\
20,35\end{array}$ & $\begin{array}{l}1,44 \\
0,66\end{array}$ & IgG & $\begin{array}{l}\text { Aappa } \\
\text { Kарра }\end{array}$ \\
\hline & 4 & & & & & \\
\hline & 5 & 1,76 & 38,80 & 1,48 & IgG & Карра \\
\hline & 6 & 10,56 & 11,00 & 2,10 & IgA & Lambda \\
\hline & 7 & 0,48 & 65,37 & 0,72 & IgG & Kappa \\
\hline & 8 & 0,28 & 63,57 & 0,40 & IgG & Kappa \\
\hline & 9 & & & & & \\
\hline & 0 & 0,38 & 19,25 & 0,58 & IgG & Kappa \\
\hline & 1 & 47,04 & 7,59 & 0,96 & IgA & Lambda \\
\hline B & 1 & 0,93 । & 57,00 & 1,40 & IgG & Карра \\
\hline & 2 & 1,76 & 18,70 & 20,52 & IgM & Lambda \\
\hline & 3 & 0,25 & 44,31 & 0,18 & IgG & Kappa \\
\hline & 4 & 0,93 & 32,78 & 37,65 & IgG & Kappa \\
\hline & & & & & IgM & Lambda \\
\hline & 5 & 35,09 & 5,28 & 0,34 & IgA & Lambda \\
\hline & 6 & 0,52 & 94,60 & 0,40 & IgG & Карра \\
\hline
\end{tabular}

Für die weiteren Untersuchungen wurden zur Isolierung und Anreicherung von Immunglobulinen jeweils 17 Seren von Patienten mit einer Gammopathie oder Dysproteinämie eingesetzt. Mit der Verwendung von Immunglobulinpräparaten insbesondere aus solchen Seren mit einem'Überschuß an monoklonalen Immunglobulinen zur Herstellung der Modellproben wird die Absicht verfolgt, über diesen Weg Immunglobulinspezifitäten mit Störeffekt aufzudecken.

In Tabelle 1 sind die eingesetzten Seren mit Gammopathie, die Klassenzugehörigkeit der Immunglobuline und ihre Leichtketten-Typisierung aufgeführt. Bei den Patienten mit Dysproteinämien bestand eine polyklonale Vermehrung der $\gamma$-Globulinfraktionen in der Träger-Elektrophorese und Immunelektrophorese (Tab. 1; Block A; Nr. 1, 4, 9).

In Tabelle 2 sind die Ergebnisse aus Modellproben ohne Digoxinzusatz wiedergegeben. Hier wurden fragliche Digoxinmengen zwischen $0,27-0,6 \mathrm{nmol} / \mathrm{l}$ gemessen. Die Werte liegen an der unteren Nachweisgrenze oder darunter. Diese Ergebnisse wurden zu einer Gruppe zusammengefaßt, weil nach einem Zusatz von Digoxin zu diesen Modellproben Digoxin-vortäuschende Effekte in Abhängigkeit vom Im-

Tab. 2. Gemessene Dixoginmengen in Modellproben mit unterschiedlicher Zusammensetzung der Probenmatrix. Nr. 1-6 identifizieren die Patientenseren 1-6 aus Tab. 1, Gruppe B, aus denen die in den Proben eingesetzten Immunglobuline isoliert und angereichert wurden. Die Mengenzusätze zu den Proben werden dargestellt durch

$\mathrm{a}=0 \mathrm{~g} / \mathrm{l} ; \quad \mathrm{d}=35 \mathrm{~g} / \mathrm{l}$

$\mathrm{b}=15 \mathrm{~g} / \mathrm{l} ; \quad \mathrm{e}=50 \mathrm{~g} / \mathrm{l}$.

$c=25 \mathrm{~g} / \mathrm{l}$

$\mathrm{r}=$ Korrelations-Koeffizient; $\mathrm{t}=\overline{\mathrm{x}}_{\mathrm{D}} / \mathrm{S}_{\overline{\mathrm{x}}_{0}} ;($ für $\mathrm{t}$-Test nach Student $) \mathrm{t}=\sqrt{\frac{\mathrm{r}^{2}(\mathrm{~N}-2)}{1-\mathrm{r}^{2}}}$ (für $\mathrm{t}-\overline{\mathrm{T}}^{3}$ est bei Korrelationen); $\mathrm{p}=$ Wahrscheinlichkeitsgrad. $a / b ; a / c ; a / d ; b / c ; c / d ; d / e=$ gepaarte Daten für den $t-T e s t$.

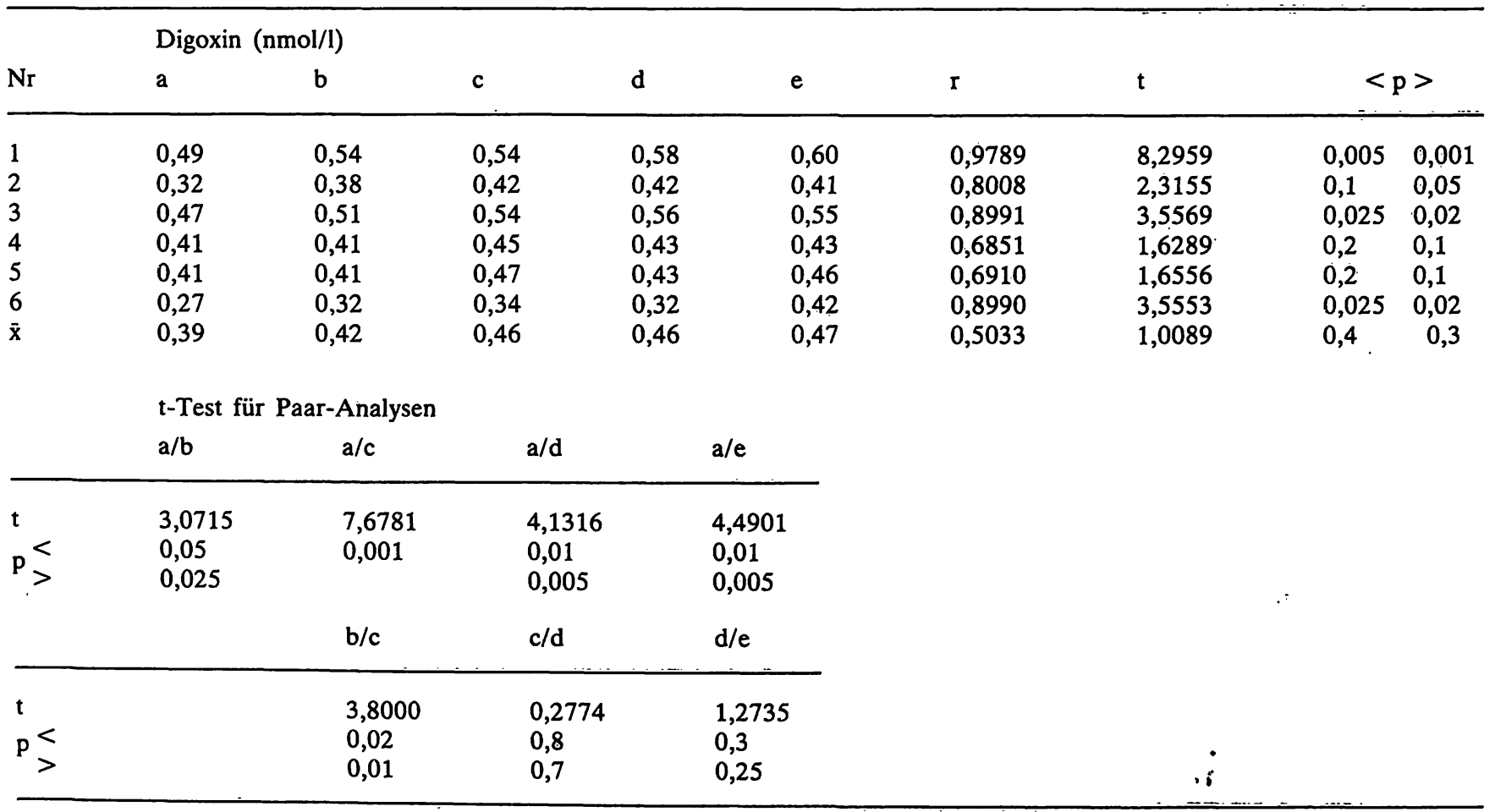


munglobulingehalt der Proben nicht nachweisbar waren (Tab. 3). Die Seren, aus denen die den Proben zugesetzten Immunglobuline isoliert wurden, sind in Tabelle 1 in der Gruppe B zusammengefaßt. Ein Anstieg von Digoxinmengen in Abhängigkeit steigender Immunglobulinmengen läßt sich nur in der Seriennummer 1 statistisch sichern (Tab. 2). Werden die gefundenen Meßdaten in Tabelle 2 entsprechend dem Immunglobulingehalt nach den Gruppen a, b, c, d, e zusammengefaßt, ergibt sich die Möglichkeit, die Unterschiede in den gemessenen Digoxinmengen zwischen den Gruppen auf Signifikanz mit dem t-Test für Paar-Analysen nach Student zu prüfen. Danach sind zwischen den Gruppen a und b, sowie den Gruppen b und c die gemessenen aber fälschlicherweise ansteigenden Digoxinmengen signifikant. Eine weitere Zunahme der Immunglobulinmengen in den Proben entsprechend den Gruppen $d$ und e führt dann aber zu keinem weiteren Anstieg der Meßwerte (Tab. 2).

Proben gleicher Matrix (Immunglobulinpräparate aus Patientenseren der Gruppe B) zeigen bei der Erweiterung der Versuchsanordnung durch Digoxinzusatz keine Abhängigkeit der Digoxinvortäuschung vom Immunglobulingehalt (Tab. 3). Von Serie zu
Serie war der Zusatz von Digoxin verschieden, innerhalb der Serie aber von Probe zu Probe gleich. Die hier von Probe zu Probe in der Serie feststellbaren Schwankungen der wiedergefundenen Digoxinmengen sind willkürlich. Die Berechnung der Wiederholbarkeit der gemessenen Digoxinmengen in der Serie ergab im Sinne der statistischen Präzisionskontrolle sogar vertretbare Variationskoeffizienten (Tab. 6).

Diesen geschilderten Ergebnissen können die, die in den Tabellen 4 und 5 dargestellt sind, gegenübergestellt werden. Hier dienten zum Herstellen der Immunglobulinpräparate Patientenseren, die in der Gruppe A der Tabelle 1 aufgeführt sind. In allen Proben, deren Ergebnisse in Tabelle 4 wiedergegeben sind, wurden wiederum fragliche Digoxinmengen im Bereich der unteren Nachweisgrenze oder darunter gemessen. In den Serien Nr. 4, 7, 9, 10 und 11 konnte eine Abhängigkeit zwischen vorgetäuschten Digoxinmengen und dem Immunglobulingehalt der Probe statistisch gesichert werden. Zwischen den Gruppen $a$ und $b$ ist nach dem $t$-Test für Paar-Analysen die Digoxinvortäuschung in Abhängigkeit vom Immunglobulingehalt ebenfalls signifikant (Tab. 4).

Tab. 3. Gemessene Digoxinmengen in Modellproben nach Digoxinaufstockung mit jeweils gleichen Mengen pro Probe in einer Serie ae, aber unterschiedlichen Mengen von Nr. 1-6. Zusammensetzung der Probenmatrix: Nr. 1-6 identifiziert die Patientenseren 1-6 aus Tab. 1, Gruppe B, aus denen die Immunglobuline, die den Proben zugesetzt wurden, isoliert und angereichert wurden. Die Mengen an Immunglobulinzusätzen zu den Proben werden dargestellt durch

$\mathrm{a}=0 \mathrm{~g} / \mathrm{l} ; \quad \mathrm{d}=35 \mathrm{~g} / \mathrm{l}$

$\mathrm{b}=15 \mathrm{~g} / \mathrm{l} ; \quad \mathrm{e}=50 \mathrm{~g} / \mathrm{l}$.

$c=25 \mathrm{~g} / \mathrm{l}$

$\mathrm{r}=$ Korrelations-Koeffizient; $\mathrm{t}=\overline{\mathrm{x}}_{\mathrm{D}} / \mathrm{S}_{\overline{\mathrm{x}}_{0}} ;($ für $\mathrm{t}$-Test nach Student $) \mathrm{t}=\sqrt{\frac{\mathrm{r}^{2}(\mathrm{~N}-2)}{1-\mathrm{r}^{2}}}$ (für $\mathrm{t}-$ Test bei Korrelationen); $\mathrm{p}=$ Wahrscheinlichkeitsgrad. $a / b ; a / c ; a / d ; b / c ; c / d ; d / e=$ gepaarte Daten für den t-Test.

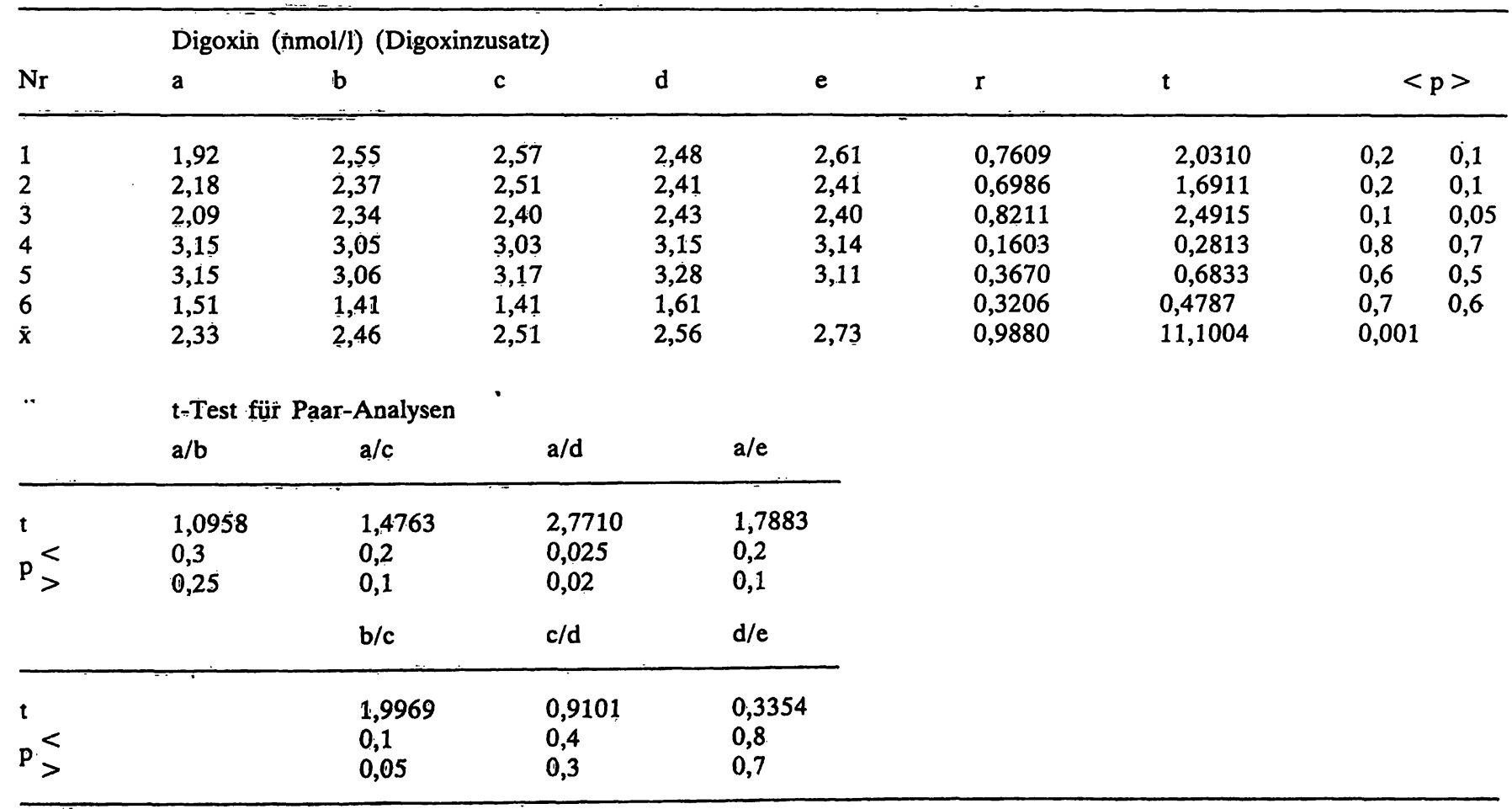


Tab. 4. Gemessene Digoxinmengen in Modellproben mit unterschiedlicher Zusammensetzung der Probenmatrix. Nr. 1-11 identifizieren die Patientenseren 1-11, aus Tab. 1, Gruppe A, aus denen die in den Proben eingesetzten Immunglobuline isoliert und angereichert wurden. Dic Mengenzusätze zu den Proben werden dargestellt durch

$\begin{array}{ll}\mathrm{a}=0 \mathrm{~g} / \mathrm{l} ; & \mathrm{d}=35 \mathrm{~g} / \mathrm{l} ; \\ \mathrm{b}=15 \mathrm{~g} / \mathrm{l} ; & \mathrm{e}=50 \mathrm{~g} / \mathrm{ll} .\end{array}$

$\mathrm{c}=25 \mathrm{~g} / \mathrm{l}$;

$\mathrm{r}=$ Korrelations-Koeffizient; $\mathrm{t}=\overline{\mathrm{x}}_{\mathrm{D}} / \mathrm{S}_{\overline{\mathrm{x}}_{\mathrm{D}}} ;($ für $\mathrm{t}-$ Test nach Student $) \mathrm{t}=\sqrt{\frac{\mathrm{r}^{2}(\mathrm{~N}-2)}{1-\mathrm{r}^{2}}}$ (für $\mathrm{t}-$ Test bei Korrelationen); $\mathrm{p}=$ Wahrscheinlichkeitsgrad. $a / b ; a / c ; a / d ; b / c ; c / d ; d / e=$ gepaarte Daten für den t-Test.

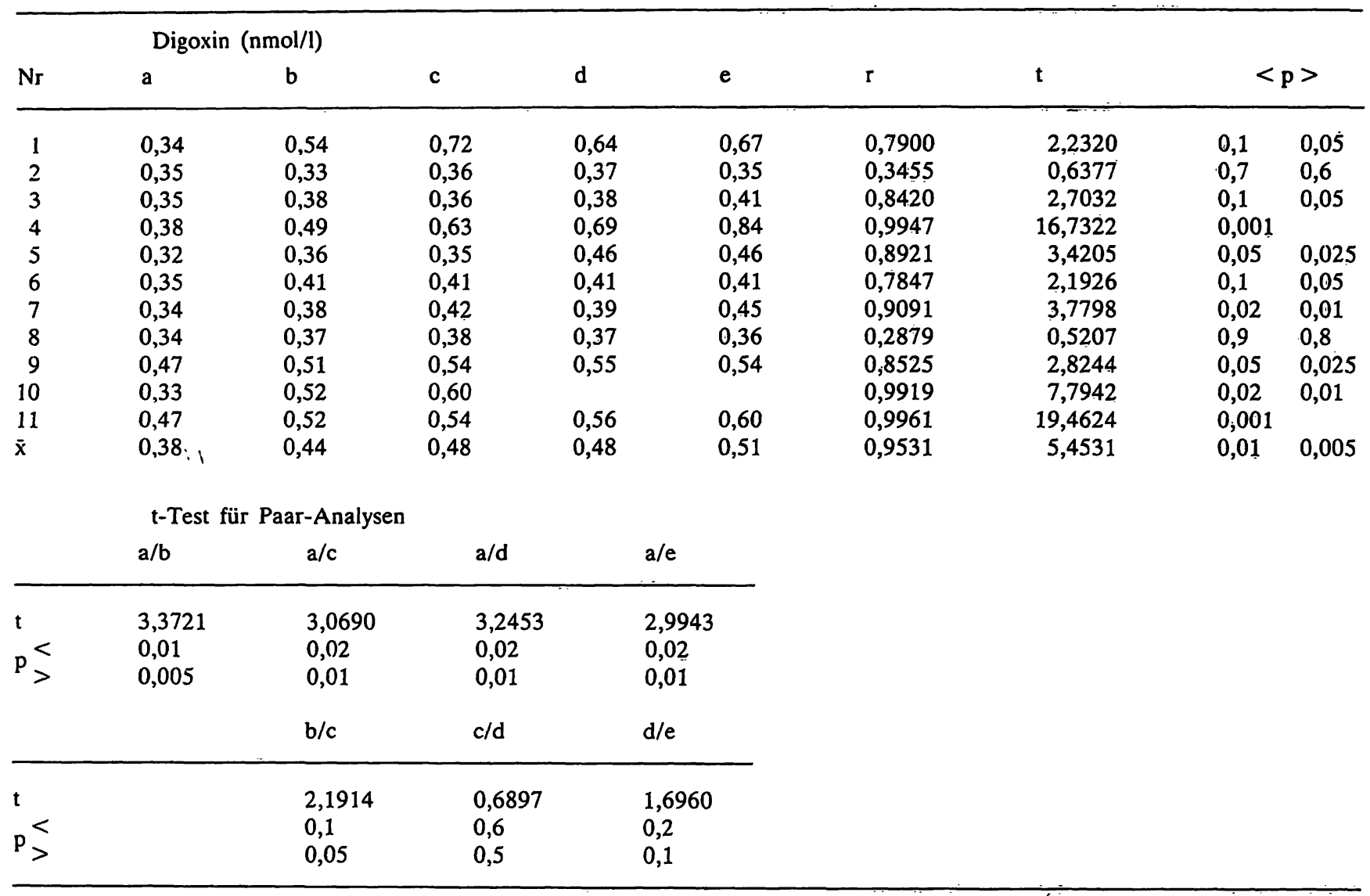

Die Digoxinwiederfindung wird nach Zusatz von Digoxin zu den Modellproben, die Immunglobuline aus den Patientenseren der Gruppe A Tabelle 1 enthielten, deutlich gestört. Auch hier waren die Digoxinzusätze in den einzelnen Proben einer Serie wieder gleich, von Serie zu Serie aber verschieden. In diesen Modellproben besteht zwischen den Immunglobulinmengen und der vorgetäuschten Digoxinmenge eine signifikante Abhängigkeit. Mit steigenden Immunglobulinmengen in der Probe wird zunehmend Digoxin vorgetäuscht (Tab. 5).

Auch der Rheumafaktor kann im Enzym-Test ${ }^{\circledR}$ Digoxin interferieren und falsch zu hohe Digoxinwerte vortäuschen. Aus einem solchen Serum wurde die Rheumafaktor-Aktivität nach einer Vorschrift von Kolb (5) isoliert und angereichert. In einem Digoxin-haltigen Serum vermag der Zusatz dieses Rheumafaktors Digoxinmengen vorzutäuschen. Die quantitative Abhängigkeit ist auch hier statistisch belegt (Tab. 7).

\section{Diskussion}

Proteine interferieren im heterogenen enzymimmunologischen Verfahren Enzymun-Test ${ }^{\circledR}$ Digoxin. Es bewirken steigende Proteinmengen eine Abnahme der Absorbanz. Dieser Effekt wird sowohl mit Proteinspezies der Albumin- als auch der Immunglobulinfraktion des Serums erhalten (Ab. 1, 2). Sofern die Proteinmatrix der Standardproben der der Serumproben entspricht, werden solche unspezififischen Reaktionen kompensiert. Quantitative Veränderungen in den Gesamtproteinmengen haben dann keinen Digoxin vortäuschenden Effekt, wenn die Proben entweder kein Digoxin oder Mengen im Bereich der unteren Nachweisgrenze enthalten. Die testspezifische Funktion zwischen Menge und Meßsignal (Absorbanz bei $405 \mathrm{~nm}$ ) hat zur Folge, daß unspezifische Reaktionen bei hohen Absorbanzwerten für das Meßergebnis nicht relevant werden (Abb. 1, 2). 
Tab. 5. Gemessene Digoxinmengen in Modellproben nach Digoxinaufstockung mit jeweils gleichen Mengen pro Probe in einer Serie ae, aber unterschiedlichen Mengen von Nr. 1-11. Zusammensetzung der Probenmatrix: Nr. 1-11 identifiziert die Patientenseren 1-11, aus Tab. 1, Gruppe A, aus denen die Immunglobuline, die den Proben zugesetzt wurden, isoliert und angereichert wurden. Die Mengen an Immunglobulinzusätzen zu den Proben werden dargestellt durch

$\mathrm{a}=0 \mathrm{~g} / \mathrm{l} ; \quad \mathrm{d}=35 \mathrm{~g} / \mathrm{l}$

$\mathrm{b}=15 \mathrm{~g} / \mathrm{l} ; \quad \mathrm{e}=50 \mathrm{~g} / \mathrm{l}$.

$\mathrm{c}=25 \mathrm{~g} / \mathrm{l}$

$r=$ Korrelations-Koeffizient; $t=\bar{x}_{D} / S_{\bar{x}_{D}} ;($ für $t-T e s t$ nach Student $) t=\sqrt{\frac{r^{2}(N-2)}{1-r^{2}}}$ (für $t-T e s t$ bei Korrelationen); $p=$ Wahrscheinlichkeitsgrad. $a / b ; a / c ; a / d ; b / c ; c / d ; d / e=$ gepaarte Daten für den $t-T e s t$.

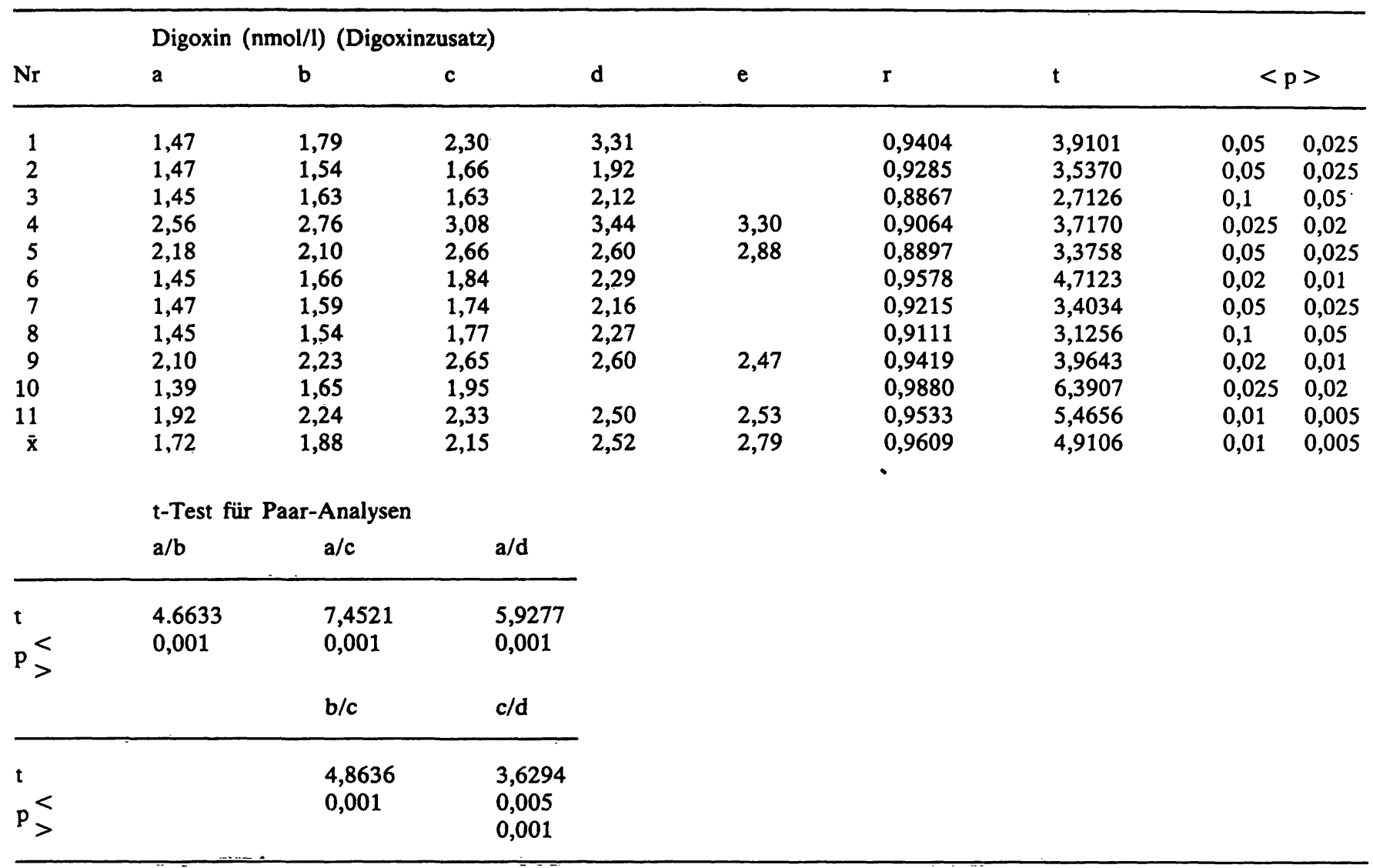

Tab. 6. Variationskoeffizient in der Wiederholbarkeit der gemessenen Digoxinwerte B in den Serien Nr. 1-6 und den Serienlängen a-e der Tab. 3 und $A$ in den Serien der Nr. 1-11 und der Serienlänge $a=e$ der Tab. 5 .

\begin{tabular}{rrrr}
\hline A & & B & \\
$\mathrm{Nr}$ & $\mathrm{VK}(\%)$ & $\mathrm{Nr}$ & $\mathrm{VK}(\%)$ \\
\hline & 36,2 & 1 & 11,0 \\
1 & 10,0 & 2 & 5,1 \\
2 & 16,8 & 3 & 6,0 \\
3 & 13,0 & 4 & 1,9 \\
4 & 12,0 & 5 & 2,6 \\
5 & 19,7 & 6 & 6,5 \\
6 & 17,3 & & \\
7 & 20,9 & & \\
8 & 9,8 & & \\
9 & 16,9 & & \\
10 & 10,7 & & \\
11 & & & \\
\hline
\end{tabular}

Tab. 7. Rheumafaktor-Titer bestimmt nach RheumaTec ${ }^{\circledR}, \mathrm{Zu}-$ satz von Rheumafaktoraktivitäten (Titermengen) zu einem digoxinhaltigen Serum; statistische Beurteilung der Abhängigkeiten:

$r=$ Korrelations-Koeffizient; $t=\sqrt{\frac{r^{2}(N-2)}{1-r^{2}}}$
$(t-T e s t$ bei Korrelationen);

$\mathbf{N}=$ Anzahl der Untersuchungspaare;

$\mathrm{p}=$ Wahrscheinlichkeitsgrad.

\section{Rheumafaktor}

(Titer)

Digoxin

(nmol/l)

\begin{tabular}{lll}
\hline 0 & & 1,92 \\
$1: 3$ & & 2,15 \\
$1: 60$ & & 2,30 \\
$1: 100$ & & 2,56 \\
$1: 130$ & 2,62 \\
$1: 160$ & & 2,82 \\
& & \\
& $r=0,9928$ & \\
& $t=16,5494$ & \\
& $N=6$ & \\
\hline
\end{tabular}


Dennoch werden in gewissen Serumproben klinisch nicht plausible, offenbar falsch zu hohe Digoxinmengen gemessen. Nach eigenen Beobachtungen handelt es sich dabei vornehmlich um Serumproben von Patienten mit Gammopathie. Im Rahmen solcher Erkrankung kann zum Beispiel die unbegrenzte Vermehrung eines Zellklons zu übermäßiger Produktion einer Immunglobulinspezifität führen. Sie wird als Ursache der falschen Meßwerte in Betracht kommen. In solchen Fällen weicht die Probenmatrix von der Standardmatrix erheblich ab.

Es liegt zunächst der Verdacht nahe, daß quantitative Abweichungen in den Proteinmengen der Probenmatrix gegenüber der Standardmatrix die Ursache für falsche Meßergebnisse sind. Es wäre aber auch denkbar, daß es Immunglobulinspezifitäten im menschlichen Serum gibt, die eine besondere Effektivität in diesem Test haben und deshalb als Störquelle in Betracht kommen. Unspezifische Aggregation mit dem wandständigen Antikörper und/oder Anlagerung an die Gefäßwand des ReaktionsgefäBes einer solchen Immunglobulinspezifität hätte eine sterische Behinderung wandständiger Antikörper mit Verlust der Bindungsfähigkeit für spezifische Antigene zur Folge. Dies führt zur Vortäuschung falsch zu hoher Digoxinmengen in der Probe.

Um Immunglobulinspezifitäten mit solchen Nebenwirkungen aufzudecken, wurden aus Seren mit monoklonaler Immunglobulinvermehrung oder Dysproteinämie (Tab. 1) die Immunglobuline präparativ dargestellt und mit Albumin zu Modellproben rekombiniert. Unter Aufrechterhaltung gleichbleibender Gesamtproteinmengen in den so hergestellten Proben wurden die Mengenverhältnisse der Proteinfraktionen in den aus jeweils einer Serumpräparation hergestellten Modellproben variiert (Tab. 2, 3, 4, 5). Damit bestanden in der Zusammensetzung der Proteinmatrix zwischen den Modellproben und den Standardproben erhebliche Unterschiede, aber die Gesamtproteinmenge in den Proben blieb unverändert.

Die aus den Modellproben ermittelten Ergebnisse lassen zunächst erkennen, daß selbst bei groben Veränderungen der Zusammensetzung der Proteinmatrix der Proben ein zu erwartender Einfluß auf die Meßergebnisse durch das Kompensationsprinzip des Verfahrens weitgehend vermieden wird (Tab. 2, $3 \mathrm{u}$. 4). Sofern die Modellproben kein Digoxin enthielten (Tab. 2, 4), lagen die gefundenen Meßwerte im Bereich der unteren Nachweisgrenze oder auch darun- ter. Die erheblichen Unterschiede in der Zusammensetzung der Probenmatrix bei unverändert bleibenden Gesamtproteinmengen der Proben waren ohne systematischen Einfluß auf die Meßwerte.

Den Modellproben in den Tabellen 2 und 4 wurden in einem zweiten Versuch verschiedene Digoxinmengen zugesetzt (Tab. 3, 5). Die erheblichen Verschiebungen in der Proteinmatrix hatten wieder in einem Teil der Modellproben (Tab. 3) keinen Einfluß auf die Meßergebnisse. Sie werden über die Standardmatrix kompensiert. Demgegenüber zeigen die in Tabelle 5 zusammengefaßten Ergebnisse eine mathematisch-statistisch beweisbare Abhängigkeit der Meßwerte von der Proteinmatrix. Die hier bei der Zubereitung der Modellproben benutzten Immunglobuline (Tab. 1, Block A) führen zu einem über die Standardmatrix nicht mehr kompensierbaren Verlust reaktionsfähiger wandständiger Antikörper im Probengefäß. Der Effekt, die Vortäuschung von Digoxin, ist in diesen Proben in seinem Ausmaß abhängig von der zugesetzten Immunglobulinspezifität und ihrer Menge. Die unspezifische Reaktion der Immunglobuline wird für das Ergebnis relevant, wenn die Probe zusätzlich Digoxin enthält. Mit steigenden Mengen dieser Immunglobuline in diesen Proben werden entsprechend falsch zu hohe Digoxinmengen angezeigt (Tab. 5). Da diese Immunglobuline aus Serum von Patienten mit Gammopathie oder Dysproteinämie präpariert wurden, kann davon ausgegangen werden, daß es im menschlichen Serum Immunglobulinspezifitäten gibt, die eine besondere Aggregationsneigung zum wandständigen Antikörper haben oder über eine besondere Affinität zum Wandmaterial des Probengefäßes verfügen. Im Rahmen einer Gammopathie können solche Proteine mit den geschilderten Eigenschaften vermehrt im Blut auftreten und kämen dann als wirksame Störquelle in diesem Test in Betracht.

In diesen Zusammenhängen steht auch der Effekt des $\bar{R}$ heumafaktors, der durch unspezifische Aggregation mit den wandständigen Antikörpern zu sterischen Behinderungen in der Antigen-Antikörperreaktion führt mit entsprechender Abnahme der Bindung enzymmarkierter Antigene und der Vortäuschung falsch zu hoher Digoxinmengen (Tab. 7).

Es ist zu vermuten, daß die in der vorliegenden Arbeit diskutierten Störmöglichkeiten für jeden Immunoassay, der auf dem Kompetitionsprinzip beruht, gelten. Der Enzymun-Test ${ }^{\circledR}$ Digoxiñ steht hier nur exemplarisch. 


\section{Literatur}

1. Pope, R. M. \& McDuffy, S. I. (1981) J. Lab. Clin. Med. 97, 842-853.

2. Henney, C. S. (1969) Ann. N. Y. Acad. Sci. 168, 52-61.

3. Kusubawa, R., Gato, M., Yoschida, H. \& Yoschida, T. (1977) Int. Arch. Allergy Appl. Immunol. 54, 247-258.

4. Ziegelmaier, R. \& Bicker, R. (1982) Laboratoriumsblätter $32,35-39$.

5. Kolb, G. (1983) Eine neue Methode zur Adsorption und Isolation von Rheumafaktoren; Dissertation Marburg.

6. Schultze, H. E. \& Gross, H. (1929) Biochem. Z. 215, 115127.

7. Kekwick, R. A. (1940) Biochem. J. 34, 1248-1254.

8. Persönliche Mitteilung von Dr. Mattersberger, Boehringer Mannheim, Tutzing.

9. Mancini, G., Carbonara, H. O. \& Heremans, I. F. (1965) Immunochemistry 2, 235-254.

10. Weichselbaum, T. E. (1946) Amer. J. Clin. Pathol. 10,4049.

11. Lothar Sachs (1983) Angewandte Statistik, pp. 242 ff. Springer-Verlag Berlin, Heidelberg, New York, Tokyo.

12. Munz, E., Kessler, A., Koller, P. U. \& Busch, E. N. (1979) Lab. Med. 3, 71-76.

Priv. Doz. Dr. med. Rolf Allner

Chefarzt Zentrallabor Städt. Kliniken

Pacelliallee 4

D-6400 Fulda 


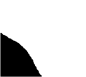

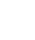

